\title{
Z-Plasty: useful uses in dermatologic surgery*
}

\author{
Hugo Barreiros ${ }^{1}$
}

\author{
João Goulão ${ }^{1}$
}

DOI: http://dx.doi.org/10.1590/abd1806-4841.20142298

\begin{abstract}
Z-Plasty is a procedure involving the transposition of two interdigitating triangular flaps. This technique is useful in a number of dermatological procedures. It can be used to correct defects resulting from surgical complications. This paper highlights several examples where Z-Plasty is of benefit in dermatosurgery, including the correction of: ectropion, lip misalignment and bifid earlobe. A valuable technique that every dermatologist should be able to perform.
\end{abstract}

Keywords: Ambulatory surgical procedures; Surgery, plastic; Surgical flaps; Surgical procedures, minor

Z-Plasty is a procedure which involves the transposition of two interdigitating triangular flaps. This transposition leads to a gain in length along the direction of the common limb of the $\mathrm{Z}$ and the change of its direction. ${ }^{1}$ Z-Plasty is an extremely useful procedure in this respect. The technique was first introduced in 1856 by Denonvilliers to correct a lower lid ectropion. ${ }^{2}$ Surgeons later proceeded to use it to correct contractures of the oral commissure, axillary burn synechiae, and joint mobility hindered by cicatricial bands.

Symmetry is one of the basic principles. ${ }^{1}$ The traditional Z-Plasty is designed to ensure that the length of both the lateral and central limbs and the angles created by their junction are equal. The length of the limbs can vary depending on circumstances, but it is essential for them to be equal. The flaps are typically designed with angles ranging from 30 to 75 degrees (Figure 1). Angles of less than 30 degrees may incur tip necrosis, and angles of over 75 degrees create flaps that are difficult to rotate, creating dog-ears as well as increased tension. Variations in the traditional Z-Plasty that involve unequal flap angles may be needed in the reconstruction of certain defects.

This valuable technique can be very helpful in several simple dermatologic procedures. One example is the reconstruction of the infra-orbitary region.
Very often the surgical removal of a tumour in this region can potentially lead to an ectropion as a complication. While multiple methods exist to correct this defect, one of the simplest is Z-Plasty. A number of factors need to be taken in consideration: the central limb axis of the $\mathrm{Z}$ should be positioned in the scar; big angles lead to a big gain in length and a big change in the direction of the scar; $\mathrm{Z}$ limbs should never exceed $1 \mathrm{~cm}$ in the face since this will occasion a poor cosmetic result; big scars should be treated with multiple ZPlasty. ${ }^{3}$ Another common situation where Z-Plasty can be helpful is the correction of lip misalignment. ${ }^{4,5}$ Normal anatomy can be easily restored by realigning tissues produced by the transposition of the two triangular flaps, with an excellent cosmetic outcome (Figure 2). Cicatricial bands can result from several dermatologic procedures. Correction is very simple

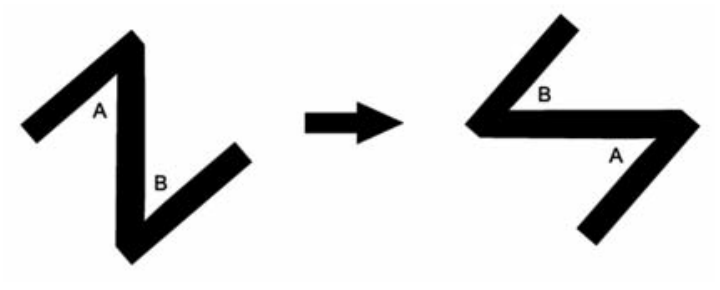

Figure 1: The Z-Plasty concept

Financial Support: None

Physicians, Hospital Garcia de Orta - Almada, Portugal 

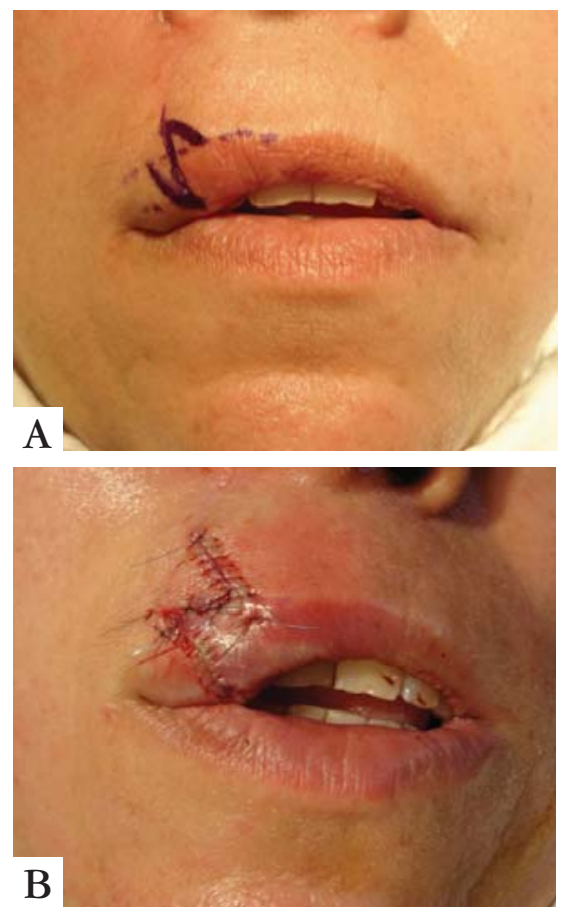

Figure 2: Upper lip misalignment correction. (A) This poor cosmetic outcome resulted from a previous dermatologic surgery. (B) Easy restoration of tissues anatomy can be achieved using ZPlasty

\section{REFERENCES}

1. McGregor AD, McGregor IA. The Z-plasty. In: McGregor AD, McGregor IA. Fundamental techniques of plastic surgery and their surgical applications. 10th ed. New York: Churchill Livingstone; 2000. p. 21-34.

2. Denonvilliers CP. Blepharoplastice. Bull Soc Chir. 1856;7:213-7.

3. Shockley WW. Scar revision techniques: z-plasty, w-plasty, and geometric broken line closure. Facial Plast Surg Clin North Am. 2011;19:455-63.

4. Taher M, Bennett R. Revision of upper lip vermilion border elevation. Dermatol Surg. 2007;33:225-8.

5. Wentzell JM, Lund JJ. Z-plasty innovations in vertical lip reconstructions. Dermatol Surg. 2011;37:1646-62.

6. Sokol JA, Schwarcz RM. A better way to repair torn earlobes using a modified z-plasty. Dermatol Surg. 2011;37:1506-8.

7. McLaren LR. Cleft earlobe: A hazard of wearing earrings. $\mathrm{Br} \mathrm{J}$ Plast Surg. 1954;7:162-5. using Z-Plasty. Our final example is the treatment of the bifid earlobe. ${ }^{6}$ Lengthening of a pre-existing earlobe hole is a common result of the use of heavy ear jewellery. Simple suture does not work because the hole is covered by epithelium. Removing the ephitelium and suturing the defect was proposed in 1954 by McLaren, but this technique results in a lack of tissue support that is incompatible with the use of earrings. ${ }^{7}$ Several repair methods have been described in the literature and Z-Plasty is one of them. Transposition flaps offer good support, and six months after surgery it is possible to use earrings again.

The versatility and simplicity of Z-Plasty is confirmed by its long history of success. It is a valuable technique that every dermatologist should be able to perform given that it can resolve common complications of dermatologic surgery. Z-Plasty has been and continues to be an important tool in a surgeon's armoury.

\author{
MAILING ADDRESS: \\ Hugo Barreiros \\ Avenida Torrado da Silva, \\ Pragal \\ 2801-951 Almada \\ Portugal \\ E-mail: hbarreiros@gmail.com
}

How to cite this article: Barreiros H, Goulão J. Z-Plasty: useful uses in dermatologic surgery. An Bras Dermatol. 2014;89(1):187-8. 